\title{
The Electrical Performance of Polyamide 66/Poly(vinylidene fluoride) with Vinyl Acetate-Maleic Anhydride Copolymer
}

\author{
Rui Li, ${ }^{1}$ Zhiliang Shi, ${ }^{1}$ Dongliang Kuang, ${ }^{2}$ and Jianzhong Pei ${ }^{1}$ \\ ${ }^{1}$ Highway School, Chang'an University, Xi'an 710064, China \\ ${ }^{2}$ School of Materials Science and Engineering, Chang'an University, Xian 710064, China \\ Correspondence should be addressed to Jianzhong Pei; peijianzhong@126.com
}

Received 21 August 2015; Revised 19 December 2015; Accepted 21 December 2015

Academic Editor: Bernabé Luis Rivas

Copyright (c) 2016 Rui Li et al. This is an open access article distributed under the Creative Commons Attribution License, which permits unrestricted use, distribution, and reproduction in any medium, provided the original work is properly cited.

\begin{abstract}
The electric performance of the PA66/PVDF blends filled with various amount of copolymer synthesized from vinyl acetate-maleic anhydride (VAMA) was investigated. PA66/VAMA/PVDF blends show high dielectric constants, low dielectric loss, and excellent breakdown strength, which were important indexes in the actual application of dielectric material. The VAMA copolymer improves the dielectric and piezoelectric performance of the PA66/PVDF blends. Meanwhile, the addition of VAMA obviously decreases the dielectric loss and breakdown strength of the blends. PA66/PVDF blends filled with 3 wt\% VAMA exhibited the best electric ability. The stable dielectric constants of the all-polymeric blends can be tuned by adjusting the content of the VAMA. The created all-polymeric blends represent a novel dielectric material that is technologically simple and easy to process forward application for flexible electronics.
\end{abstract}

\section{Introduction}

With the continuous development of science and technology, the need of high performance material urges people to open up the composites, which display their advantages outweighing their components, and also it is hard for single polymer to meet requirements [1-3]. Polyvinylidene fluoride (PVDF) and Polyamides (PA) are two famous polar polymers. In particular, $\mathrm{PVDF}$ is known as polymer composites materials characterized by excellent dielectric and piezoelectric properties $[4,5]$. However, in the actual application, the high cost of PAll and the high melting point $\left(220 \sim 240^{\circ} \mathrm{C}\right)$ of PA66 limited the applications in many fields. Also there are still few studies about PA66/PVDF blends. In addition, the PA66/PVDF blends are an interesting system because of some specific interactions [6-8]. As a new material, a blend of PA66 and PVDF offers a strong potential for the application in the field of capacitor [9]. According to the property index requirements of energy storage material, the polymers selected should have two features: (1) the polymer itself should be partly crystallized and (2) the molecular chain of the polymer should have polar groups. This makes the polymer have a more sophisticated orientation under the action of electric field. While improving the dielectric constant of polymer, it can be used as energy storage material with the inherent low breakdown strength [10]. Therefore, VAMA offers a strong potential in the application of the allpolymer blends [11].

The purpose of this work is to investigate the dielectric and piezoelectric performance of the PA66/PVDF blends filled with various amount of VAMA. Firstly, the copolymer VAMA from acetic acid vinyl ester and maleic anhydride was synthesized. Through grafting reaction can lower the melting point of PA66 and the PA66/PVDF blends obtained from PA66/VAMA/PVDF by melt blending through torque rheometer. It is expected that this research can be useful in promoting the applications of the PA66/PVDF blends under oil-free lubrication conditions.

\section{Experimental}

2.1. Materials. PVDF is from Shanghai $3 F$ Material Co., Ltd., $T_{m}=175^{\circ} \mathrm{C}$, and $d=1.75 \mathrm{~g} / \mathrm{cm}^{3}$. PA66 is from Sinopec Baling Branch Co., Ltd., $T_{m}=225^{\circ} \mathrm{C}$, and $d=1.15 \mathrm{~g} / \mathrm{cm}^{3}$. The related products are commercially available. 
<smiles>C=C(C)OC(C)=O</smiles>

FIGURE 1: Reaction scheme of VAc and MAH copolymerization.

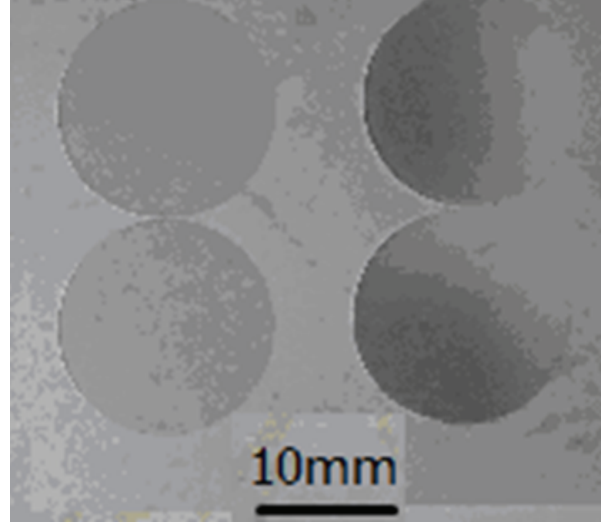

(a)

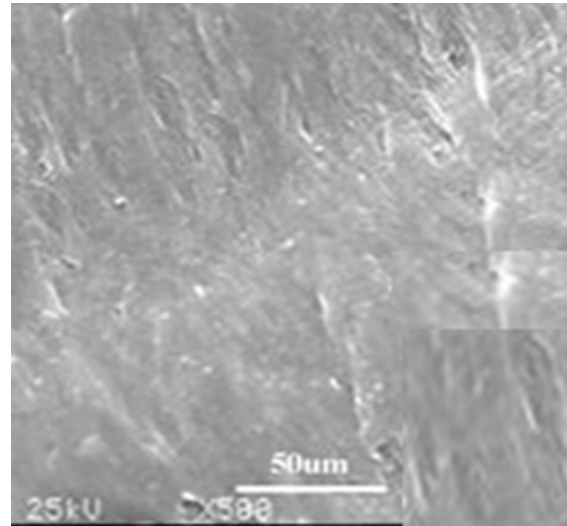

(b)

FIgURE 2: The photograph of the physical films.

2.2. Synthesis of Graft Copolymer of Vinyl Acetate-Maleic Anhydride. The methylbenzene was added to a three-mouth flask, which was equipped with stirrer, thermometer, and reflux condensing tube, and then the maleic anhydride was added and dissolved into methylbenzene slowly after stirring and heating. After the solution turns to be transparent, acetic acid vinyl ester is added. Also, $1.4 \%$ initiator azodiisobutyronitrile was added. The temperature is controlled at $90^{\circ} \mathrm{C}$. The copolymer is subsided for $3 \mathrm{~h}$, filtered, and washed with dichloroethane for 5 times, removing the unreacted maleic anhydride. After vacuum drying under $60^{\circ} \mathrm{C}$, white powdered graft copolymer can be obtained [12]. Figure 1 is the reaction scheme of VAc and MAH copolymerization.

2.3. Preparation of PA66/VAMA/PVDF. PA66, VAMA, and PVDF were mixed for $45 \mathrm{~min}$ based on different volume ratios in torque rheometer, and then press vulcanizer was used to press the evenly pressed PA66/VAMA/PVDF blends into thin plate. In addition, microtablet machine is used to press plate into around $1 \mathrm{~mm}$. Finally, the samples were polished and silvered by silver paint.

2.4. Instrumental Analysis. The microstructures of blends were characterized by scanning electron microscopy (SEM) and Fourier transform infrared spectroscopy (FTIR). The electric properties of the samples were performed by HIOKI3532-50 LCR; the frequency is from $10^{2}$ to $10^{6} \mathrm{~Hz}$ at room temperature.



Figure 3: FTIR spectrum of PA66/VAMA/PVDF.

\section{Results and Discussions}

3.1. Structure Analysis. The photographs of the films were shown in Figure 2(a). It can be seen from Figure 2(b) that PA66/PVDF blends present a unique structure of ordered arrangement which is evenly distributed. The infrared spectrum of PA66/VAMA/PVDF was tested by Fourier transformation infrared spectrometric analyzer. It can be seen from Figure 3 that the absorption peak where the wavenumber of $1780 \mathrm{~cm}^{-1}$ was the stretching vibration of carboxylic acid $\mathrm{C}=\mathrm{O}$ and $1374 \mathrm{~cm}^{-1}$ was the flexural vibration of methyl. 


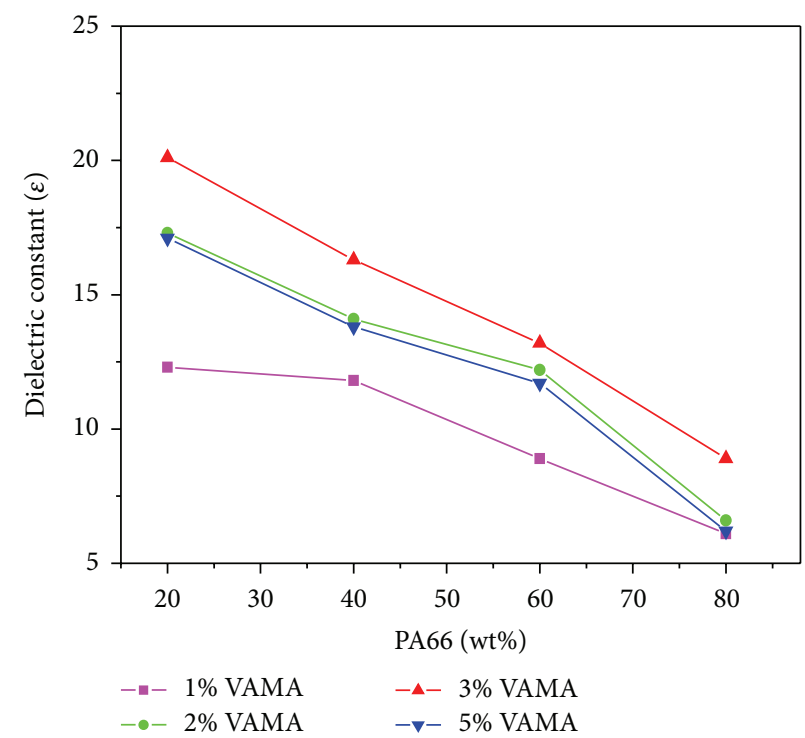

(a)

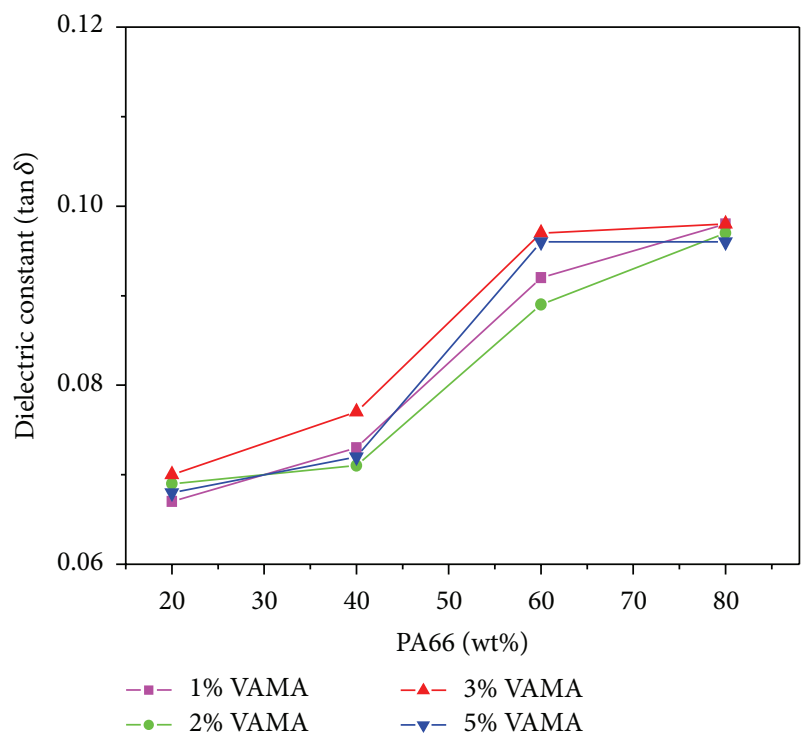

(b)

FIGURE 4: Variation of $\varepsilon$ and $\tan \delta$ with VAMA content.

The strength of the absorption band of $1314 \mathrm{~cm}^{-1}$ was greater than that of $1433 \mathrm{~cm}^{-1}$, which is the significant characteristic of vinyl acetate structure, which proves the reaction of copolymerization between acetic acid vinyl ester and maleic anhydride. Also, it can be found that the two acid anhydride peaks disappear at $1780 \mathrm{~cm}^{-1}$ and $1740 \mathrm{~cm}^{-1}$. When the infrared spectral curve of PA66/VAMA/PVDF was compared with that of copolymer VAMA, it can be found that PA66/VAMA/PVDF characteristic peak of cyclic amide disappears at $1414 \mathrm{~cm}^{-1}$, which shows that the anhydride of VAMA molecule has chemical reaction with -NH of PA66.

3.2. Dielectric Properties. At room temperature, when the frequency is $10^{3} \mathrm{~Hz}$, the dielectric properties of PA66/VAMA/ PVDF blends are obtained for analysis after adding different mass fractions of VAMA ( $1 \mathrm{wt} \%, 2 \mathrm{wt} \%, 3 \mathrm{wt} \%$, and $5 \mathrm{wt} \%$ ) as shown in Figure 4. The quantities of VAMA added are those by which it can improve not only the dielectric constant of PA66/PVDF blends that have been improved but also the dielectric loss of blends that have been down. It proved that VAMA can make polar dipoles easier to be oriented within PA66/PVDF blends, thus lowering the dielectric loss of blends. The relation curve of the dielectric constants between the PA66/VAMA/PVDF blends and the content of VAMA was shown in Figure 4. Obviously, the influence of copolymer VAMA on the dielectric constant of PA66/VAMA/PVDF blends is $3 \mathrm{wt} \%>2 \mathrm{wt} \%>5 \mathrm{wt} \%>1 \mathrm{wt} \%$. When PA66 and PVDF were melted and blended, adding VAMA can improve the molecules flexibility of PA and PVDF, and also the dielectric constant of PA66/VAMA/PVDF can be improved. However, excessive VAMA was prone to the grafting ratio of PA66, which is not good for the collection and delivery of the system charge. Therefore, the results indicate that, after grafting, a distinct layer structure is formed

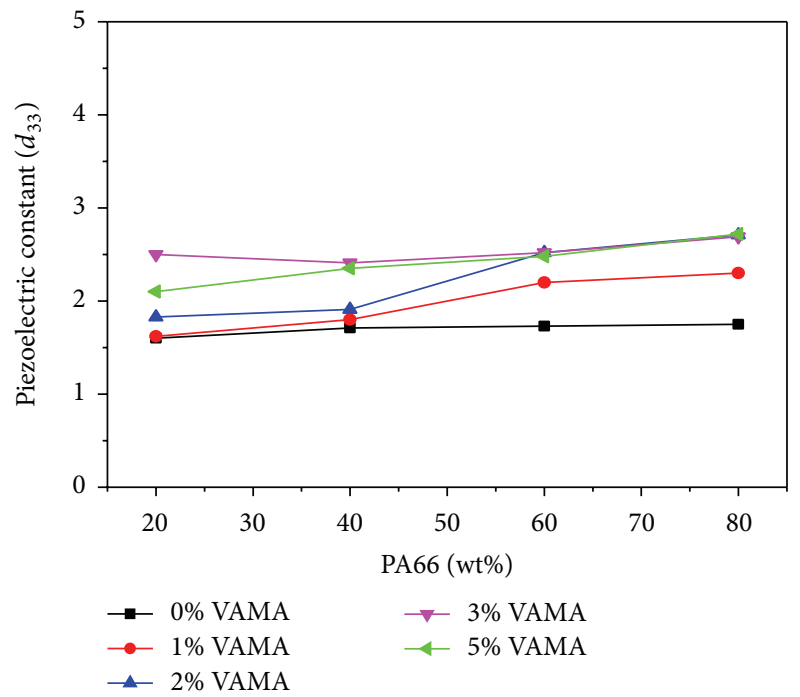

Figure 5: Piezoelectric constant $d_{33}$ of PA66/PVDF blends.

in PA66/VAMA/PVDF blends. The optimum content of the VAMA was $3 \%$.

3.3. Piezoelectric Properties. The PA66/PVDF blends with $0 \mathrm{wt} \%, 1 \mathrm{wt} \%, 2 \mathrm{wt} \%, 3 \mathrm{wt} \%$, and $5 \mathrm{wt} \%$ VAMA were chosen for studying piezoelectric coefficient $\left(d_{33}\right)$ owing to the highest dielectric constant and good interfacial compatibility. Figure 5 shows the relationship between the piezoelectric constant $d_{33}$ and the different VAMA and PA66 content. Although the samples do not exhibit super piezoelectric properties, PA66/VAMA/PVDF blends have a better enhancement in the piezoelectric constant $d_{33}$ than that of the PA66/PVDF at room temperature. The results show 


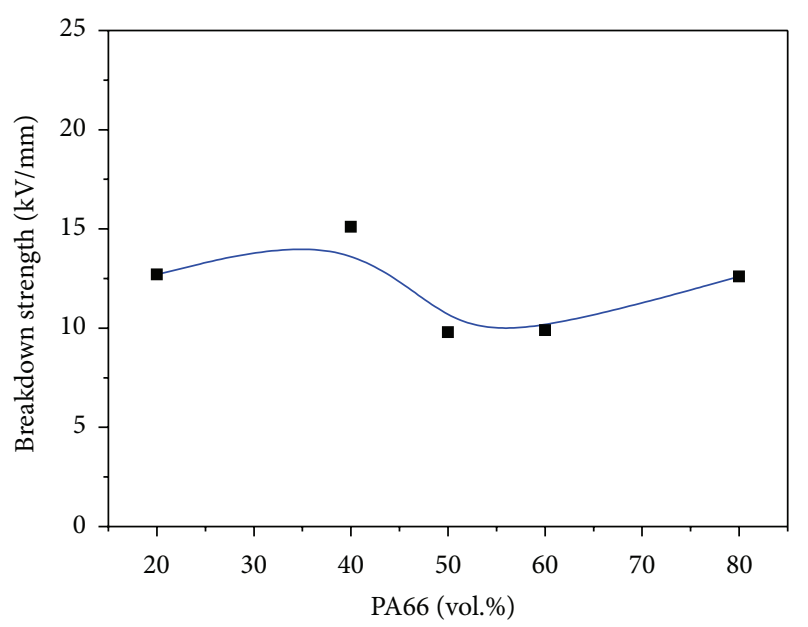

FIgURE 6: Breakdown strength of PA66/VAMA/PVDF (3 wt\% VAMA).

that the polarization degrees of PA66/VAMA/PVDF were enhanced by VAMA so as to improve the piezoelectric properties.

3.4. Breakdown Strength. Breakdown strength is an important index in the actual application of dielectric material. Regarding the material structure, there are many factors which influence the breakdown strength, which are distribution uniformity of crystal particle, crystal size, and the material defects such as internal air holes. In addition, to varying degrees, the shape of samples, thickness, and the shape of electrode can also influence the final breakdown strength. In order to reduce the influence of material structure on the breakdown strength, samples which were $1 \mathrm{~mm}$ thick were selected in this experiment.

The breakdown strength of PA66/VAMA/PVDF blends with PA66 volume fraction increase was shown in Figure 6. When the volume of PA66 was 40\%, PA66/VAMA/PVDF blends reach the maximum breakdown strength $\left(E_{b}=\right.$ $15.3 \mathrm{kV} / \mathrm{mm}$ ). And when the volume content of PA66 was $60 \mathrm{vol} . \%$, the breakdown strength of the material has the minimum $\left(E_{b}=9.9 \mathrm{kV} / \mathrm{mm}\right)$. This is because the two polymer phases (PA66 and PVDF) within PA66/VAMA/PVDF samples were not bonded well (PA66: PVDF $=60: 40)$, which cause a low breakdown strength.

Combining the dielectric property test result of PA66/VAMA/PVDF blends, the sample with PA66: PVDF = $20: 80$ has the best dielectric properties. In order to study the influence of thickness on the breakdown strength of samples, PA66/VAMA/PVDF blends were applied to prepare different thicknesses of samples to have breakdown experiment in the volume ratio of PA66: PVDF $=20: 80$. Figure 7 is the relational curve between breakdown strength and blends thickness. It can be found from it that, with the increase of thickness, the breakdown strength declines sharply. When the thicknesses were increased to $2.0 \mathrm{~mm}$ from $0.5 \mathrm{~mm}$, the breakdown strength declined from $11.9 \mathrm{kV} / \mathrm{mm}$ continuously. Obviously, the thickness of samples has a significant influence



Figure 7: Breakdown strength of PA66/VAMA/PVDF.

on the breakdown strength. The reason is because the defects in thicker blends than in thin blends. Thereby, the breakdown strength of blends can be decreased greatly. On the other hand, due to the fringe effect of the electric field intensity, the increase of thickness strengthens the electric field for few times on the fringe of electrode. Consequently, the breakdown strength of PA66/VAMA/PVDF blends declined.

The result shows that it is easier for these defects to cause local electric field distortion under the same electric field and testing conditions. Thus, the probability to cause breakdown to dielectric medium is increased. Therefore, with the increase of testing thickness, the breakdown strength will be decreased sharply. Meanwhile, Kuang et al. found that the electric field distortion of plate electrode was significant by applying electric field simulation to different electrode structures, and the electric field being enhanced on the fringe of electrode makes it easier for the air holes on the surface of medium around electrode fringe to cause gas discharge, which caused breakdown to the entire medium $[12,13]$.

\section{Conclusions}

In conclusion, we have demonstrated a simple, efficient, and repeatable route to produce discontinuously all-polymer blends composed of PA66 and PVDF. The all-polymer blend films have a relatively high $\varepsilon$, low dielectric loss $\tan \delta$, and excellent storage modulus as induced by interfacial copolymer VAMA. The alternating copolymer VAMA prepared can be grafted on the molecular chain of PA66. The accession of VAMA can improve the interface adhesion of PVDF phase and PA66. The VAMA content of $1 \mathrm{wt} \%$ can improve the dielectric constant of PA66/PVDF blends, and the dielectric loss change of PA66/PVDF blends was not significant. When the quantity of VAMA added was $3 \mathrm{wt} \%$, the optimum content of VAMA was $3 \mathrm{wt} \%$. PA66/VAMA/PVDF blends can be used as flexible dielectric material.

\section{Conflict of Interests}

The authors declare that there is no conflict of interests. 


\section{Acknowledgments}

The research was supported by the National Natural Science Foundation of China (Grant nos. 51378073 and 51408048), the Key Program for International Science and Technology Cooperation Projects of Shaanxi province (Grant no. 2014KW10-03 and 2012KW-09), the China Postdoctoral Science Foundation (Grant nos. 2015M570805 and 2015M582592), the Fundamental Research Funds for the Central Universities of Chang'an University (Grant nos. 310821153502 and 310831151085), and the Research Fund for the Doctoral Program of Higher Education of China (Grant no. 20120205120010).

\section{References}

[1] Q. M. Zhang, H. F. Li, M. Poh et al., "An all-organic composite actuator material with a high dielectric constant," Nature, vol. 419, no. 6904, pp. 284-287, 2002.

[2] R. Mezzenga, J. Ruokolainen, G. H. Fredrickson et al., "Templating organic semiconductors via self-assembly of polymer colloids," Science, vol. 299, no. 5614, pp. 1872-1874, 2003.

[3] R. Li and J. Z. Pei, "High dielectric performance of polyamide 11/poly(vinylidene fluoride) blend films induced by interfacial glycidyl methacrylate," Polymer Science Series A, vol. 57, no. 6, pp. 792-798, 2015.

[4] Z.-M. Dang, Y.-H. Zhang, and S.-C. Tjong, "Dependence of dielectric behavior on the physical property of fillers in the polymer-matrix composites," Synthetic Metals, vol. 146, no. 1, pp. 79-84, 2004.

[5] Z. M. Dang, Y. H. Lin, and C. W. Nan, "Novel ferroelectric polymer matrix composites with a high dielectric constant at the percolation threshold," Advanced Materials, vol. 15, no. 19, pp. 1625-1628, 2003.

[6] I. S. Bayer, M. K. Tiwari, and C. M. Megaridis, "Biocompatible poly(vinylidene fluoride)/cyanoacrylate composite coatings with tunable hydrophobicity and bonding strength," Applied Physics Letters, vol. 93, no. 17, Article ID 173902, 2008.

[7] I. S. Bayer, A. Biswas, J. B. Szczech, E. Suhir, and M. G. Norton, "Radio frequency functional capacitors made of all-organic composites of thiourea in field-responsive polymers," Applied Physics Letters, vol. 92, no. 8, Article ID 083303, 2008.

[8] R. Li, C. X. Xiong, D. L. Kuang, L. J. Dong, and Y. A. Lei, "Polyamide11/poly (vinylidene fluoride) as novel flexible materials for capacitors," Macromolecular Rapid Communications, vol. 29, no. 17, pp. 1449-1454, 2008.

[9] C. Huang, Q. M. Zhang, J. Y. Li, and M. Rabeony, "Colossal dielectric and electromechanical responses in self-assembled polymeric nanocomposites," Applied Physics Letters, vol. 87, no. 18, Article ID 182901, 2005.

[10] A. Rehab and N. Salahuddin, "Nanocomposite materials based on polyurethane intercalated into montmorillonite clay," Materials Science and Engineering A, vol. 399, no. 1-2, pp. 368-376, 2005.

[11] C. A. Rogers, "Smart materials-systems the down of new materials ages," Intelligent Material Systems and Structure, vol. 4, no. 1, pp. 4-10, 1993.

[12] D. L. Kuang, R. Li, and J. Pei, "Polyamide 11/poly(vinylidene fluoride)/vinyl acetate-maleic anhydride copolymer as novel blends flexible materials for capacitors," Polymers, vol. 6, no. 8, pp. 2146-2156, 2014.
[13] S. C. Tjong, S. P. Bao, and G. D. Liang, "Polypropylene/montmorillonite nanocomposites toughened with SEBS-g-MA: structure-property relationship," Journal of Polymer Science Part B: Polymer Physics, vol. 43, no. 21, pp. 3112-3126, 2005. 

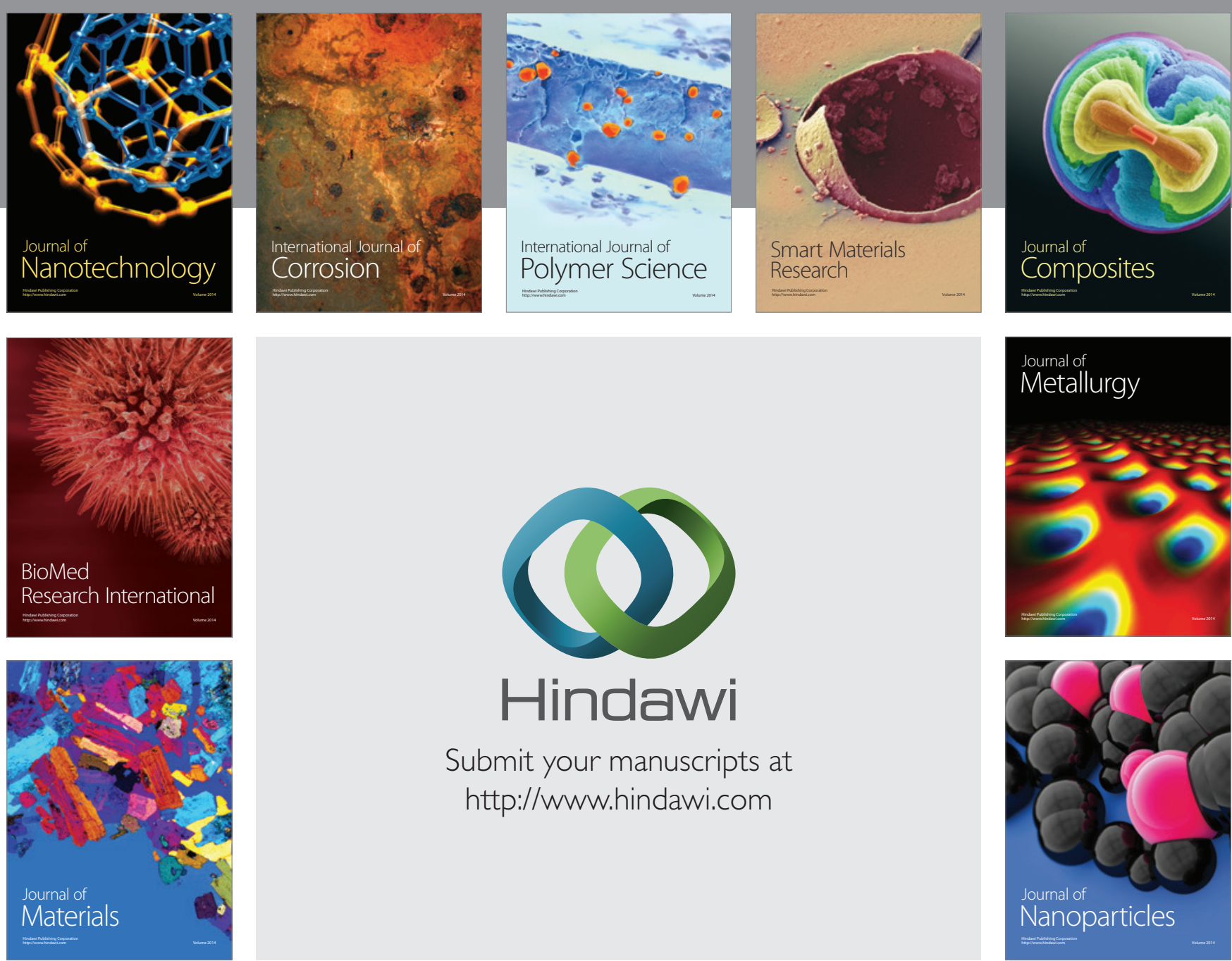

\section{Hindawi}

Submit your manuscripts at

http://www.hindawi.com

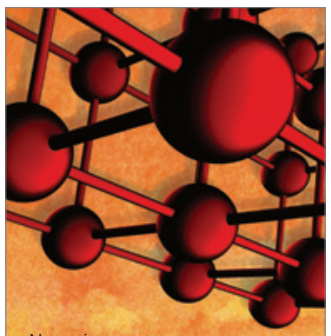

Materials Science and Engineering


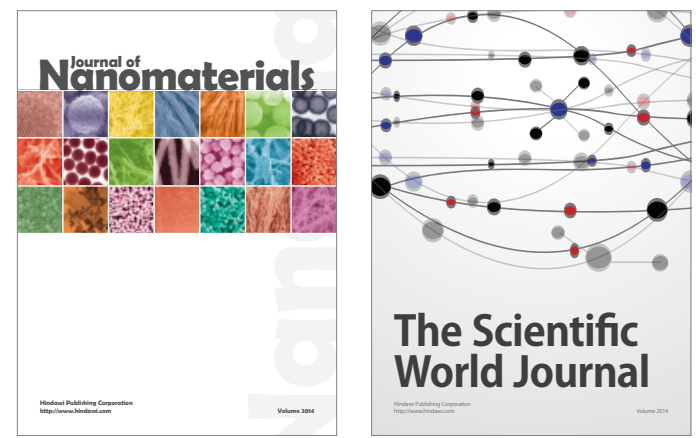

The Scientific World Journal
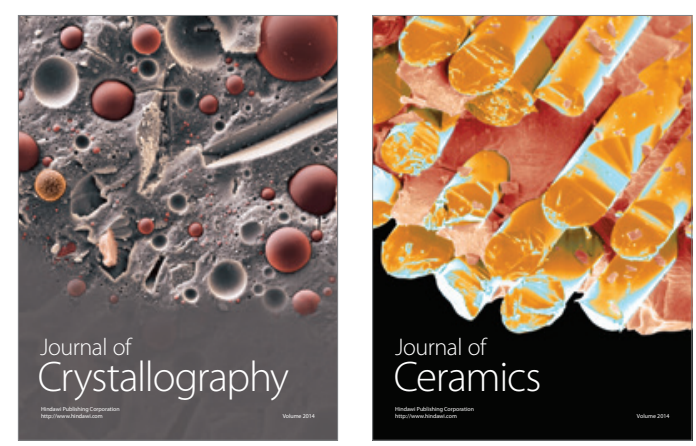
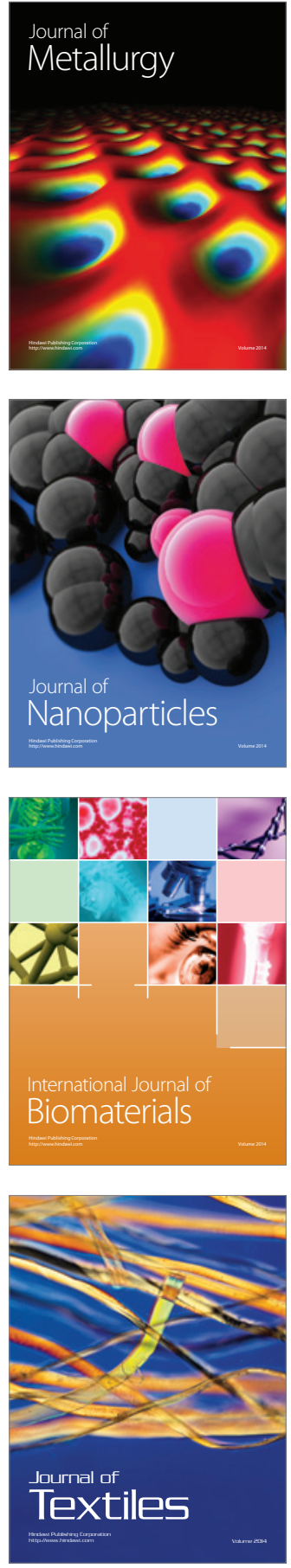